\title{
Absenteísmo de pacientes com necessidades especiais em Centros de Especialidades Odontológicas
}

\author{
Absenteeism of patients with special needs in dental specialties centers \\ Ausentismo de pacientes con necesidades especiales en centros de especialidad dental
}

Recebido: 03/03/2021 | Revisado: 10/03/2021 | Aceito: 12/03/2021 | Publicado: 20/03/2021

Thyago Campos Leite de Araújo

ORCID: https://orcid.org/0000-0003-1884-7069

Centro Universitário Dr. Leão Sampaio, Brasil

E-mail: thyagocampos2@gmail.com

Regiane Cristina do Amaral

ORCID: https://orcid.org/0000-0002-9191-0960 Universidade Federal de Sergipe, Brasil

E-mail: amaralre@yahoo.com.br

Emilio Prado Fonseca

ORCID: https://orcid.org/0000-0002-6810-133X

Universidade José do Rosário Vellano, Brasil

E-mail: emiliopraddo@ig.com.br

Luciana Butini Oliveira

ORCID: https://orcid.org/0000-0002-8755-6540

Faculdade São Leopoldo Mandic, Brasil

E-mail: lucianabutini@slmandic.edu.br

\begin{abstract}
Resumo
O presente estudo teve por objetivo verificar o não comparecimento dos pacientes com necessidades especiais em três Centros de Especialidades Odontológicas Regionais (CEO-R), localizados na região do Cariri. Os dados foram obtidos através do Sistema Integrado de Gestão em Saúde do Ceará. A fim de verificar associação entre distância e número de faltas foi realizada análise de correlação de Pearson e para descrever o padrão geográfico da ocorrência de faltas, foram construídos mapas temáticos baseados nas distribuições das taxas de faltas. Ao se correlacionar as variáveis, verifica-se correlação estatisticamente significativa para distância e percentual de faltas $(\mathrm{p}=0.003)$ distância e pacientes agendados $(\mathrm{p}=0.025)$, distância e pacientes confirmados $(\mathrm{p}=0.018)$ e distância e tratamentos concluídos $(\mathrm{p}=0.016)$. Pode-se concluir que a as faltas dos pacientes não ocorre de modo sazonal e que a distância até o CEO pode ser um preditor que apresentou correlação positiva e forte associação com o absenteísmo dos pacientes.
\end{abstract}

Palavras-chave: Pacientes desistentes do tratamento; Odontologia; Saúde pública.

\begin{abstract}
The present study aimed to verify the absence of patients with special needs in three Regional Dental Specialties Centers (CEO-R), located in the Cariri region. The data were obtained through the Integrated Health Management System in Ceará. In order to verify the association between distance and number of absences, Pearson's correlation analysis was performed and to describe the geographic pattern of the occurrence of absences, thematic maps were built based on the distributions of the absentee rates. When correlating the variables, there is a statistically significant correlation for distance and percentage of absences $(p=0.003)$ distance and scheduled patients $(p=0.025)$, distance and confirmed patients $(\mathrm{p}=0.018)$ and distance and completed treatments $(\mathrm{p}=0.016)$. It can be concluded that patients 'absences do not occur seasonally and that the distance to the CEO can be a predictor that presented a positive correlation and strong association with patients' absenteeism.
\end{abstract}

Keywords: Patient dropouts; Dentistry; Public health.

\section{Resumen}

El presente estudio tuvo como objetivo verificar la no asistencia de pacientes con necesidades especiales en tres Centros Regionales de Especialidades Dentales (CEO-R), ubicados en la región de Cariri. Los datos se obtuvieron a través del Sistema Integrado de Gestión Sanitaria de Ceará. Para verificar la asociación entre la distancia y el número de ausencias, se realizó el análisis de correlación de Pearson y para describir el patrón geográfico de la ocurrencia de ausencias, se construyeron mapas temáticos basados en las distribuciones de las tasas de ausencias. Cuando las variables están correlacionadas, existe una correlación estadísticamente significativa para la distancia y el porcentaje de ausencias $(\mathrm{p}=0.003)$ distancia y pacientes programados $(\mathrm{p}=0.025)$, distancia y pacientes confirmados $(\mathrm{p}=0.018)$ y distancia completa y tratamientos $(\mathrm{p}=0,016)$. Se puede concluir que las ausencias de los pacientes no ocurren 
estacionalmente y que la distancia al CEO puede ser un predictor que mostró una correlación positiva y una fuerte asociación con el absentismo de los pacientes.

Palabras clave: Pacientes que abandonaron el tratamiento; Odontología; Salud pública.

\section{Introdução}

O Ministério da Saúde no ano de 2004 criou o programa Brasil Sorridente, integrante da Política Nacional de Saúde Bucal (PNSB), com o objetivo de garantir ações de promoção, prevenção e recuperação da saúde bucal da população brasileira, sendo o maior programa público de saúde bucal do mundo (Brasil, 2013).

De acordo com a Portaria no 1.341, de 13 de junho de 2012, os Centro de Especialidades Odontológicas (CEOs), deverão disponibilizar no mínimo 40 horas semanais para atendimento exclusivo à pessoa com deficiência, a portaria define um valor adicional do incentivo financeiro de custeio mensal aos CEOs aos municípios que aderirem à Rede de Cuidados à Pessoa com Deficiência (Brasil, 2012).

O governo do estado do Ceará com o programa de expansão e fortalecimento da atenção Especializada à saúde implementou os Centro de Especialidades Odontológicas Regionais (CEO-R). Foi adotado o modelo de consórcio público de saúde, esse modelo permite aos municípios, a formação de parceria, para obtenção de maior ganho de escala e melhorar a capacidade técnica, gerencial e financeira na prestação de serviços públicos, congregando como entes consorciados o estado e os municípios de cada região de saúde, sendo assim um município polo, prestará assistência odontológica aos demais municípios pertencentes a sua Coordenadoria Regional de Saúde- CRES. O Contrato de Programa exige uma Programação Pactuação Consorciada - PPC, de acordo com as demandas, necessidades e perfil epidemiológico da população, os municípios rateiam financeiramente valores (Ceara, 2010).

Os CEOs são planejados para atender a determinado número de pacientes encaminhados da atenção primária. A falta dos pacientes no atendimento gera prejuízo financeiro, os custos aumentam devido à ociosidade dos recursos disponíveis e uma perda de oportunidade para oferecer o atendimento odontológico a outro Paciente com Necessidade Especial (PNE) que buscam atendimento, gerando um aumento na fila de espera. As razões que levam os pacientes a faltarem ao atendimento agendado não são bem definidas, a literatura tem mostrado que as principais causas para um aumento do absenteísmo dos pacientes em consultas, está relacionada à distância entre o município de origem, alto custo de transporte, esquecimento (Fonseca et al., 2018; Chaves et al., 2011). A maior parte dos estudos sobre o absenteísmo dos pacientes a consulta especializada não apresentam dados sobre esse não comparecimento ao atendimento agendado dos PNEs.

Dessa forma, diante dessa lacuna, o objetivo deste estudo foi investigar a porcentagem de modificação mensal (PMM) de não comparecimento dos PNEs em três Centros de Especialidades Odontológicas Regionais (CEO-R), localizados na região do Cariri sul do estado do Ceará, bem como associar a distância do município como motivo da falta destes pacientes.

\section{Metodologia}

A pesquisa foi submetida ao comitê de ética e pesquisa e aprovado (CAAE:99343418.1.0000.5374).

Trata-se de um estudo transversal, com fonte de dados obtidos através do Sistema Integrado de Gestão em Saúde do Ceará (SIGES), dados secundários, com análise quantitativa. Para o presente estudo foi utilizado dados dos 3 CEOs-R da região sul do Cariri-CE. A região do Cariri sul do Estado do Ceará está localizado a uma distância em média de $500 \mathrm{~km}$ da Capital Fortaleza, sendo as cidades de Brejo Santo, Crato e Juazeiro do Norte. Sendo utilizado como critérios de inclusão o tempo mínimo de três anos de implantação do CEO-R além de apresentarem o mesmo tipo de gestão, infraestrutura física e tipo de referenciamento para a atenção secundária. As marcações das consultas dos pacientes são realizadas através das centrais de regulação municipais com fluxo de referências externas emitidas a partir da atenção primária. O sistema utilizado 
para a marcação foi o Sistema de Regulação (SISREG), que é um sistema de informação on-line, disponibilizado pelo DATASUS, para o gerenciamento e a operação das Centrais de Regulação, as consultas de retorno são marcadas no próprio CEO onde o paciente está sendo atendido ou pela central de regulação municipal.

$\mathrm{O}$ presente estudo teve como enfoque os pacientes com necessidades especiais (PNE). Assim verificou-se que tratamento dos pacientes com necessidades especiais é realizado através de uma Programação Pactuada Consorciada (PPC) entre os entes consorciados (constituídos pelo estado e os municípios que compõem a regional de saúde) e a unidade de saúde CEO-R da seguinte forma: cada município tem direito a um número específico de vagas para a especialidade que levam em consideração o tamanho da população do município e os recursos humanos existentes. Assim foram analisados dados dos atendimentos realizados na especialidade PNE no ano de 2018. A partir dos relatórios de monitoramento dos atendimentos agendados dos PNEs do SIGES foram extraídos dados referentes ao número de pacientes agendados em primeira consulta e de retorno, total de faltas ao atendimento e número de tratamentos concluídos, obtidos mensalmente, envolvendo todos os atendimentos referentes ao ano de 2018. A fim de verificar a variação percentual mensal das taxas de faltas e verificar sazonalidade, usou-se análise de serie temporal, com análise de regressão Prais Winsten, que prevê correção de autocorrelação de primeira ordem. A variável dependente foi o logaritmo das taxas, e a variável foram os meses do histórico série. O cálculo da variação percentual mensal das taxas foi realizado com o seguindo fórmulas, como sugerido por Antunes e Waldman.

$$
-1+10 b=\Delta(1)
$$

Para o cálculo dos intervalos de confiança:

$$
\Delta 95 \mathrm{Cl}=-1+10(\mathrm{~b} \pm \mathrm{t} * \mathrm{se})(2)
$$

Onde "b" corresponde à taxa de crescimento anual. Os valores de "b" e desvio padrão (se) foram extraídos da análise de regressão, e o valor de "t" é fornecido pelo teste de mesa de distribuição t. A tendência de aumento, diminuição ou estagnação foi expressa como APC, com os respectivos intervalos de confiança (95\%), e consideramos como estacionária a tendência cujo coeficiente de regressão não foi diferente de zero $(\mathrm{p}>0,05)^{9}$. A análise foi realizada no programa Stata, versão 14 (Perea et al., 2018).

A fim de verificar associação entre distância e número de faltas foi realizada análise de correlação de Pearson, utilizando o programa SPSS 17.0.

A fim de descrever o padrão geográfico da ocorrência, foram construídos mapas temáticos baseados nas distribuições das taxas de faltas (Almeida \& Szwarcwald, 2012)

Os mapas foram construídos por municípios e por CEO-R em programa computacional específico com padronização da legenda em quatro estratos (Borden \& Thomas, 2009; Almeida \& Szwarcwald, 2012). Para a construção dos mapas foi utilizado uma base cartográfica eletrônica (formato shape file) do estado do Ceará com divisão territorial por municípios (polígonos) e disponibilizada pelo Instituto Brasileiro de Geografia e Estatística (IBGE). Para este estudo foi utilizado programa computacional TerraView ${ }^{\circledR}$ (versão 4.2.2) disponibilizado pelo Instituto Nacional de Pesquisas Espaciais 2010 (INPE).

\section{Resultados}

No presente estudo foram avaliadas as taxas de absenteísmo dos PNE em 3 CEOs da Região do Cariri - CE. A fim de verificar a sazonalidade das faltas dos pacientes especiais foi realizada análise de série temporal para o ano de 2018, sendo 
encontrada variação considerada estacionária para o período analisado e não demonstrando sazonalidade em meses festivos ou do período de férias escolares (Tabela 1 e Figura 1).

Figura 1 - Percentual de faltas dos CEOs da Região do Cariri - CE. 2018.

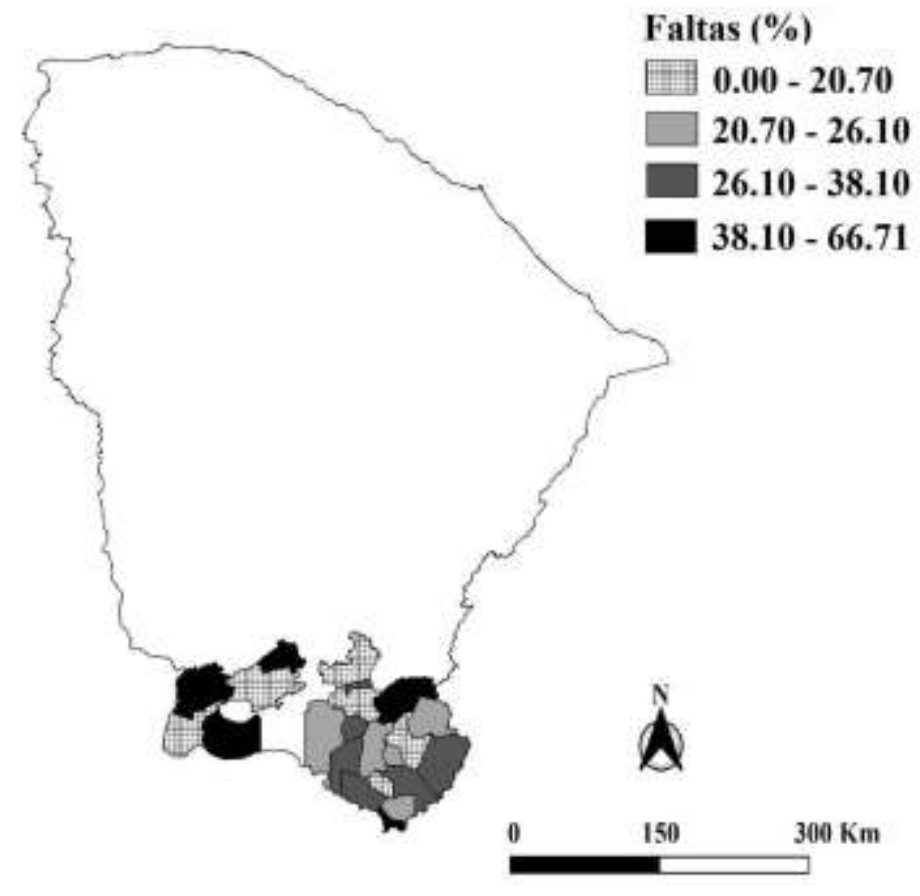

Fonte: Autores.

Tabela 1 - Percentual de mudança mensal - 2018.

\begin{tabular}{lcccc}
\hline & $\begin{array}{c}\text { (variação } \\
\text { percentual } \\
\text { mensal) }\end{array}$ & & IC & \\
\multicolumn{1}{c}{ Região } & $\mathbf{2 0 1 8}$ & Min. & Max. & Tendência \\
\hline Crato & 8.924 & -0.039 & 0.234 & Estacionária \\
Juazeiro do Norte & -4.141 & -0.117 & 0.041 & Estacionária \\
Brejo Santo & -5.207 & -0.106 & 0.005 & Estacionária \\
\hline
\end{tabular}

Fonte: Autores.

No que se refere ao número de falta dos pacientes, associado à distância do CEO sede, o município de Tarrafas que fica a $117 \mathrm{~km}$ teve $60 \%$ de faltas, o município de Aurora distante 92,6 km teve 41,8\% de faltas e Granjeiro 56,1 km teve um percentual 30\% das faltas (tabela 2). Com relação aos tratamentos concluídos totais no ano de 2018, o CEO do Crato concluiu 93\%, Juazeiro do Norte 73,8\% e Brejo Santo 39,2\%. A porcentagem de tratamento concluído foi calculada pelo número de primeiras consultas por município no ano de 2018 e número de tratamento concluído, alguns municípios concluíram tratamentos em 2018 de pacientes que estavam em tratamento de anos anteriores.

Ao se correlacionar as variáveis (correlação de Pearson) entre distancia ao CEO a sede (pelo menos 3 pacientes agendados) e percentual de faltas $(\%)$, pacientes agendados, pacientes confirmados e tratamento concluído, verifica-se 
correlação estatisticamente significativa para distância e percentual de faltas $(\mathrm{p}=0.003)$ distância e pacientes agendados ( $\mathrm{p}=0.025)$, distância e pacientes confirmados $(\mathrm{p}=0.018)$ e distância e tratamentos concluídos ( $\mathrm{p}=0.016)$ (Tabela 2). Verifica-se ainda que a maior parte dos pacientes agendados faz parte do município Sede. A Figura 1 mostra a localização dos municípios sede de CEO-R de Crato, Juazeiro do Norte e Brejo Santo e os municípios que fazem parte de cada regional de saúde.

Tabela 2 - Distância do Centro de Especialidades aos municípios regionais e percentual de faltas, número de pacientes agendados de primeira consulta, número comparecimento e número de tratamento concluídos para os pacientes especiais 2018.

\begin{tabular}{|c|c|c|c|c|c|}
\hline Município & $\begin{array}{l}\text { Distância em Km } \\
\text { da Sede do CEO }\end{array}$ & $\%$ Faltas & Agendados & $\begin{array}{l}\text { Presente na } \\
\text { Consulta }\end{array}$ & Concluídos \\
\hline Abaiara & 34.6 & 21.9 & 64 & 50 & 2 \\
\hline Araripe & 113 & 38.1 & 21 & 13 & 3 \\
\hline Assaré & 87.1 & 0 & 2 & 2 & 2 \\
\hline Aurora & 92.6 & 43.6 & 55 & 31 & 4 \\
\hline Barbalha & 10 & 34.1 & 220 & 145 & 37 \\
\hline Barro & 49.3 & 24.6 & 61 & 46 & 3 \\
\hline Brejo Santo* & 0 & 26.1 & 915 & 676 & 50 \\
\hline Crato* & 0 & 23.9 & 569 & 433 & 111 \\
\hline Campos Sales & 136 & 66.7 & 3 & 1 & 2 \\
\hline Caririaçu & 29.2 & 0 & 2 & 2 & 1 \\
\hline Granjeiro & 56.1 & 27.3 & 11 & 8 & 5 \\
\hline Jardim & 48.9 & 27.1 & 118 & 86 & 10 \\
\hline Juazeiro do Norte* & 0 & 30.6 & 1060 & 736 & 214 \\
\hline Jati & 25 & 20.7 & 82 & 65 & 7 \\
\hline Mauriti & 44.8 & 30.8 & 377 & 261 & 27 \\
\hline Milagres & 21.6 & 18.0 & 255 & 209 & 18 \\
\hline Missão Velha & 26.1 & 24.5 & 98 & 74 & 17 \\
\hline Penaforte & 38.8 & 41.3 & 80 & 47 & 7 \\
\hline Porteiras & 19.2 & 17.2 & 99 & 82 & 10 \\
\hline Salitre & 141 & 0 & 1 & 1 & 0 \\
\hline Tarrafas & 117 & 50.0 & 6 & 3 & 1 \\
\hline Várzea Alegre & 81 & 9.1 & 22 & 20 & 4 \\
\hline
\end{tabular}

*Município sede do CEO-R. ** Distância aproximada do município à sede do CEO-R em Quilômetros. Fonte: Autores.

Na Figura 2, observamos Distribuição espacial da taxa de faltas e raios de abrangência territorial a partir da sede do CEO-R, Ceará. O CEO-R de Crato possui a maior extensão territorial, enquanto que o CEO-R de Juazeiro do Norte possui a 
menor extensão territorial. O município de Campos Sales possui a maior distância em relação à sede (Crato) a que pertence, com 136 quilômetros, porém durante o ano de 2018, só teve dois atendimentos confirmados e uma falta. Entretanto, os municípios de Barbalha e Milagres, que estão localizados aproximadamente a vinte quilômetros do CEO-R sede (Juazeiro do Norte e Brejo Santo), apresentaram taxas de faltas de 25.8 e 16,4 respectivamente.

Figura 2 - Distribuição espacial da taxa de faltas e raios de abrangência territorial a partir da sede do CEO-R, Ceará.

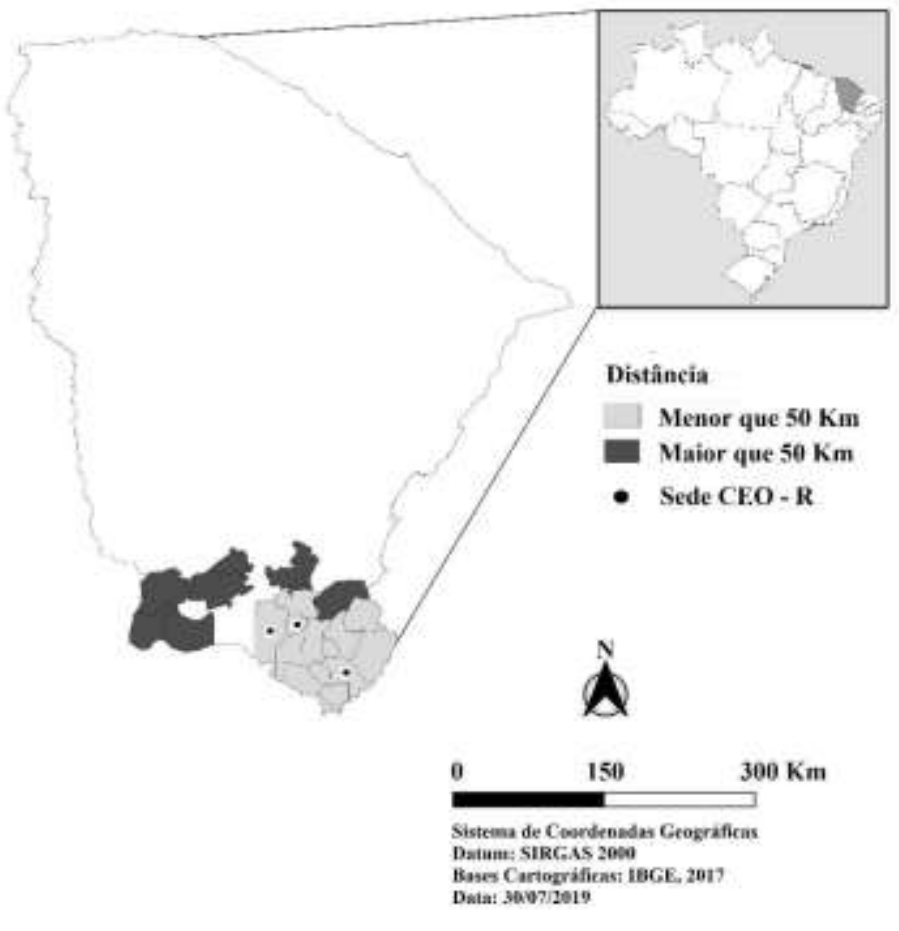

Fonte: Autores.

\section{Discussão}

Os PNEs podem apresentar condições de Saúde Bucal (SB) associada à sua alteração sistêmica, dependendo do tipo de deficiência pode torná-las mais vulneráveis as doenças bucais, o que confirma a necessidade de inclusão desse grupo populacional na atenção odontológica (Brasil, 2002). Historicamente, os PNE apresentam dificuldades de acesso aos serviços odontológicos, apesar de suas condições de saúde bucal (SB) ser desfavoráveis em relação à população em geral. A dificuldade do acesso ao atendimento odontológico dos PNE pode estar relacionada a diversos fatores, tais como a falta de conhecimento e de preparo dos profissionais da atenção básica para o atendimento a esse grupo de pacientes, negligência por parte dos responsáveis e pacientes ou desconhecimento da importância da saúde bucal desses pacientes (Macêdo et al., 2018; Couto et al., 2021).

A região do Cariri sul do estado do Ceará está a uma distância média de $500 \mathrm{~km}$ da Capital do estado Fortaleza. O estado do Ceará possui um IDH médio, porém alguns dos municípios que participaram da pesquisa possuem o IDH-baixo, como é o caso das cidades de Salitre, Caririaçu e Granjeiro. Os aspectos sociais estão relacionados a oportunidades e acesso, em suas mais variadas formas e os aspectos demográficos estão associados a características culturais e sociais, ou seja, uma pior condição social está associada a um menor acesso a medidas de prevenção, controle e tratamento de morbidades, resultando em piores condições de saúde (Sadovsky et al., 2015).

Paralelamente, os aspectos demográficos podem determinar maior capacidade de percepção dos agravos e das limitações impostas por eles (McGrath e Bedi, 2002). No que se refere ao número de falta dos pacientes, associado à distância do CEO 
sede, o município de Tarrafas que fica a $117 \mathrm{~km}$ teve $60 \%$ de faltas, o município de Aurora distante 92,6 km teve 41,8\% de faltas e Granjeiro 56,1 km teve um percentual 30\% das faltas (tabela 2). Estudo realizado para avaliar acessibilidade e motivos do absenteísmo dos pacientes no CEO de Sobral no interior do Ceará (Pinto et al., 2014), os motivos mais citados para o não comparecimento dos pacientes aos atendimentos agendados foram a dificuldade com transporte, alguns dos pacientes necessitam de transporte dos municípios para o deslocamento, a falta de recurso financeiro para condução, principalmente quando residem em municípios distantes do CEO, assemelhando com a pesquisa onde as maiores distâncias do município para o Centro foi um preditor que apresentou correlação positiva e forte associação com o aumento das faltas nas consultas, devido dependerem de transporte público municipal para consultas.

A sazonalidade pode ser definida como um movimento regular de uma série dentro de um ano (Oliveira e Favero, 2002). Conhecer este comportamento é de grande utilidade para os serviços de saúde, pois permite que adaptações e mudanças sejam feitas a partir dos dados obtidos. Uma pesquisa foi realizada para avaliar a atenção secundária em saúde bucal do CEO de Pelotas no RS, evidenciou uma queda na produção nos meses que coincidia com o período das férias escolares (Laroque et al., 2015), no estudo realizado foi encontrado uma variação considerada estacionária para o período analisado e não demonstrado sazonalidade em meses festivos, mês de romaria ou período de férias escolares. Em um mês de baixa ocupação sazonal, por exemplo, uma estratégia que o gestor poderia adotar é conceder as férias dos profissionais nesse período, caso o CEO apresente uma baixa demanda em determinado mês, compreender esta sazonalidade é crucial para desenvolver ações de planejamento eficazes para o setor, como também algumas doenças estão sujeitas à variação sazonal, com picos em determinados períodos do ano.

Um dos indicadores do Programa Nacional de Melhoria do Acesso e da Qualidade-PMAQ para atenção básica é a relação entre a quantidade de tratamentos concluídos e as primeiras consultas odontológicas programáticas. Esse indicador permite avaliar se a equipe está tendo resolutividade no acesso, de acordo com o manual do PMAQ do MS este indicador serve como base para planejamento e monitoramento do acesso e da resolubilidade do atendimento da equipe de Saúde Bucal, além de subsidiar processos de melhoria de gestão e avaliação das ações (Brasil, 2015). Na presente pesquisa, verificou-se a porcentagem de tratamentos concluídos associado com as primeiras consultas aos PNEs nos três CEOs do sul do estado do Ceará. Os municípios de Crato, Juazeiro do Norte e Brejo Santo, apresentaram respectivamente 93, 73,8 e 39,2\% de tratamentos concluídos no ano de 2018, uma das possibilidades do município de Crato atingir esse elevado número de tratamento concluído se deu pelo fato de a maioria dos PNEs assistido nesse Centro serem do mesmo município, não necessitando de grandes deslocamentos.

No município de Brejo Santo há cidades com distâncias com mais de $90 \mathrm{~km}$ e essas distancias tiveram correlação positiva com o número de tratamento concluído, quanto mais distante, menor o número de conclusão do tratamento, os atendimentos odontológicos ao PNE em grande parte necessitam de várias sessões para finalização do tratamento, muitas vezes limitados, devido às condições de saúde dos pacientes, juntamente com predisposição que esses pacientes têm de adoecer com mais facilidade, além disso, existe falta de compreensão dos seus responsáveis sobre a importância de levar os pacientes para o tratamento odontológico, as baixas condições socioeconômicas, como o custo com transporte e alimentação também podem contribuir para não conclusão do tratamento odontológico, torna-se de extrema importância o correto encaminhamento dos PNE da atenção básica para a atenção secundária, pois grande parte desses pacientes poderia ter seu tratamento realizado na ESF (Sampaio et al., 2004; Santos \& Lima, 2014).

O absenteísmo dos usuários da rede pública brasileira de saúde tem se revelado um problema crônico. O número de consultas e exames agendados e não realizados, devido ao não comparecimento dos usuários, atinge índices significativamente altos, que podem ser percebidos em todas as regiões do Brasil e em diversos tipos de atendimento e especialidade (da Cruz et 
al., 2018), em relação ao acesso dos usuários ao CEOs, considerando que a maioria dos pacientes são dependentes do SUS para atendimento, esperava-se que a utilização dos serviços especializado fosse maior.

Os resultados da pesquisa mostraram um número considerado de faltas dos pacientes durante as consultas iniciais e de retorno para conclusão do tratamento. É possível avaliar se a equipe mantém boa relação entre acesso (número de primeiras consultas odontológicas programáticas) e resolubilidade (número de tratamentos concluídos), ou seja, em que medida ela está concluindo os tratamentos iniciados, considerando a fórmula de cálculo deste indicador.

Dada a complexidade para o setor público, o deslocamento, principalmente de distancias maiores acima de $50 \mathrm{~km}$, parece, ainda, ser um grande entrave de acesso desse grupo de pacientes ao setor secundário, que necessitam de transporte público para conseguir ser atendido, associado principalmente as questões financeiras, o custo com o deslocamento, torna-se inviável para a população que possui baixa renda. Os gestores municipais devem intensificar ou criar uma política de melhoria dos transportes públicos municipais dentro das regionais de saúde, o fator distancia teve impacto direto no número de faltas desses pacientes para realização de procedimentos de caráter não urgente e emergencial.

É importante a gestão avaliar e monitorar o absenteísmo dos pacientes, entender e analisar os principais motivos do não comparecimento e as consequências dessas ausências para o setor especializado. A busca ativa desses pacientes e o contato com o cirurgião-dentista da atenção primária vão servir como base para uma tomada de decisão que levem à correção ou minimização desse absenteísmo. A ausência dos pacientes tem impacto direto na organização da demanda e financeira do setor, nesse período que o PNE está ausente, o profissional pode ficar com seu tempo ocioso, já que o paciente tem dia e hora marcada para o atendimento no CEO-R e esse não comparecimento sendo avisado previamente, poderia diminuir a fila de espera e substituição dos PNEs faltosos.

O paciente ou responsável não deve ter receio de informar ao serviço, inclusive para desmarcar consultas previamente agendadas. A utilização de aplicativos de internet poderia facilitar essa comunicação para uma confirmação ou para desmarcar $\mathrm{o}$ atendimento, otimizando o atendimento.

\section{Conclusão}

Pode-se concluir que a as faltas dos pacientes não ocorre de modo sazonal e que a distância até o CEO foi um preditor que apresentou correlação positiva e forte associação com o absenteísmo dos pacientes. Além disso, houve correlação positiva entre a distância do local do atendimento e o número de tratamento concluído. Há necessidade de futuros trabalhos que desenvolvam o tema sobre absenteísmo visto a importância quanto ao planejamento em saúde.

\section{Agradecimentos}

Aos gestores dos CEOs - Regionais da região do Cariri - Ceará.

\section{Referências}

Almeida, W. D. S. D. \& Szwarcwald, C. L.(2012). Mortalidade infantil e acesso geográfico ao parto nos municípios brasileiros. Rev Saúde Pública,46(1),6876.

Antunes, J. L. F., \& Waldman, E. A. (2002).Trends and spatial distribution of deaths of children aged 12-60 months in São Paulo, Brazil, 1980-98. Bull World Health Organ, 80(5),391-8.

Brasil. Legado Brasil [online]. 2013. http://legado.brasil.gov.br/noticias/saude/2013/10/brasil-sorridente-e-o-maior-programa-de-saude-bucal-do-mundo

Brasil. Ministério da Saúde. Portaria GM/MS no. 1.341. Cria incentivos adicionais para os CEO que fizerem parte da Rede de Cuidados à Pessoa com Deficiência, 2012, 29 jun.

Brasil. Ministério da Saúde. Portaria GM 1.060/02. Política Nacional de Saúde da Pessoa com Deficiência. Diário Oficial da Republica Federativa do Brasil, Brasília (DF); 2002. 
Research, Society and Development, v. 10, n. 3, e40310313527, 2021

(CC BY 4.0) | ISSN 2525-3409 | DOI: http://dx.doi.org/10.33448/rsd-v10i3.13527

Brasil. Qualificação dos indicadores do manual instrutivo para as equipes de atenção básica (saúde da família, saúde bucal e equipes parametrizadas) e NASF. Básica. Brasilia (DF); 2015. 103 p.

Borden, D. D. J. T. \&Thomas ,W. (2009). Hodler Cartography: Thematic Map Design. (6a ed.), 329 p.

Ceará. Secretaria da Saúde. Estatuto do Consórcio Público de Saúde da Microrregião de Cascavel - CPSMCAS. Diário Oficial do Estado, Fortaleza, Caderno Único; 2010; out $5 ;$ p.38

Chaves, S. C. L, Cruz, D. N., Barros, S. G. \& Figueiredo, A. L.(2011). Avaliação da oferta e utilização de especialidades odontológicas em serviços públicos de atenção secundária na Bahia, Brasil. Cad Saúde Pública, 27(11),143-54.

Cruz, D. F. , Cavalcanti, R. P., de Lucena, E. H. G. \& Padilha, W. W. N. (2018). A regulação da atenção à saúde bucal e o absenteísmo: cenários e possibilidades. Rev Família, Ciclos de Vida e Saúde no Contexto Social, 6(2),228-37.

Couto, G. R., Santo, M. A. L., Paiva, S. M., Prado, E. F. \& Amaral, R. C. (2021). Análise de desempenho da atenção odontológica especializada em rede de cuidados a pessoa com necessidades especiais. Research, Society and Development, 10 (2), e35710212678.

Fonseca, E. P., Silva, J. P. D .J., Vedovello, S. A. S., Souza, L. Z., Pereira, A. C. \& Meneghim, M. C.(2018) Factors associated with absences from orthodontic treatment at a dental specialty center. Cien Saude Colet,23(1),287-94.

IBGE. Instituto Brasileiro de Geografia e Estatística. Bases cartográficas do Brasil. IBGE; 2010.

INPE .Instituto Nacional de Pesquisas Espaciais. Programa Comuputacional TerraView ® [ www.inpe.gov,br.]

Laroque, M. B., Fassa, A. G. \& Castilhos, E. D.(2015). Avaliação da atenção secundária em saúde bucal do Centro de Especialidades Odontológicas de Pelotas, Rio Grande do Sul, 2012-2013. Epidemiol Serv Saúde,24(3),421-30.

Macêdo, G. L., Lucena, E. E. S., Lopes, I. K. R. \& Batista, L. T. O. (2018). Acesso ao atendimento odontológico dos pacientes especiais: a percepção de cirurgiões-dentistas da atenção básica. Rev Ciênc Plural,4(1),67-80.

McGrath, C. \& Bedi, R. (2002). Population based norming of the UK oral health related quality of life measure (OHQoL-UK). Br Dent J,193(9):521-4.

Oliveira, M. A. \& Favero, L. P. L. (2002). Uma breve descrição de algumas técnicas para análise de séries temporais: Séries de Fourier, Wavelets, Arima, Modelos Estruturais para séries de tempos e redes neurais. São Paulo: EdUSP.

Perea, L. M. E., Pere,s M. A., Boing, A. F. \& Antunes, J. L. F. (2018). Tendência de mortalidade por câncer de boca e faringe no Brasil no período $2002-2013$. Rev Saúde Pública,52:10.

Pinto, V. P. T., Teixeira, A. H., Santos, P. R., Araújo, M. W.A ., Moreira, M. A. G. \& Saraiva, S. R. M.(2014). Avaliação da acessibilidade ao Centro de Especialidades Odontológicas de abrangência macrorregional de Sobral, Ceará, Brasil. Ciênc Saúde Coletiva,19(7),2235-44.

Sadovsky, A. D. I., Poton, W. L., Reis-Santos, B., Barcelos, M. R. B. \& Silva, I. C. M.(2015). Índice de Desenvolvimento Humano e prevenção secundária de câncer de mama e colo do útero: um estudo ecológico. Cad Saúde Pública,31(7),1539-50.

Sampaio, E. F. C., Neves, F. \& Martins, M. G. A.(2004). Perfil odontológico dos pacientes portadores de necessidades especiais atendidos no Instituto de Previdência do Estado do Ceará. Rev Bras Promoç Saúde,17(3),127-34.

Santos, C, \& Lima, M.(2014). Perfil epidemiológico dos pacientes com necessidades especiais atendidos em um centro de especialidades odontológicas do interior baiano. Rev Baiana de Saúde Pública,38(1),83. 